\title{
Is the Internet a useful and relevant source for health and health care information retrieval for German cardiothoracic patients? First results from a prospective survey among 255 Patients at a German cardiothoracic surgical clinic Dietrich Stoevesandt ${ }^{1}$ and Claudius Diez*2
}

\author{
Address: ${ }^{1}$ Department of Radiology, Martin-Luther-University Halle-Wittenberg, Ernst-Grube-Str. 40, D-06097 Halle/Saale, Germany and \\ ${ }^{2}$ Department of Cardiothoracic Surgery, Martin-Luther-University Halle-Wittenberg, Ernst-Grube-Str. 40, D-06097 Halle/Saale, Germany \\ Email: Dietrich Stoevesandt - dietrich@stoevesandt.com; Claudius Diez* - claudius.diez@medizin.uni-halle.de \\ * Corresponding author
}

Published: 21 October 2006

Journal of Cardiothoracic Surgery 2006, I:36 doi: 1 0.1 I86/I 749-8090-I-36

Received: 20 July 2006

Accepted: 21 October 2006

This article is available from: http://www.cardiothoracicsurgery.org/content/l/l/36

(C) 2006 Stoevesandt and Diez; licensee BioMed Central Ltd.

This is an Open Access article distributed under the terms of the Creative Commons Attribution License (http://creativecommons.org/licenses/by/2.0), which permits unrestricted use, distribution, and reproduction in any medium, provided the original work is properly cited.

\begin{abstract}
Background: It is not clear how prevalent Internet use among cardiopathic patients in Germany is and what impact it has on the health care utilisation. We measured the extent of Internet use among cardiopathic patients and examined the effects that Internet use has on users' knowledge about their cardiac disease, health care matters and their use of the health care system.
\end{abstract}

Methods: We conducted a prospective survey among 255 cardiopathic patients at a German university hospital.

Results: Forty seven respondents ( $18 \%)$ used the internet and 8,8 \% $(n=23)$ went online more than 20 hours per month. The most frequent reason for not using the internet was disinterest $(52,3$ $\%)$. Fourteen patients $(5,4 \%)$ searched for specific disease-related information and valued the retrieved information on an analogous scale ( $I=$ not relevant, $5=$ very relevant $)$ on median with 4,0 . Internet use is age and education dependent. Only $36(14,1 \%)$ respondents found the internet useful, whereas the vast majority would not use it. Electronic scheduling for ambulatory visits or postoperative telemedical monitoring were rather disapproved.

Conclusion: We conclude that Internet use is infrequent among our study population and the search for relevant health and disease related information is not well established.

\section{Background}

Several international studies suggested that more than half and as much as $80 \%$ of adults with Internet access use it for health care purposes $[4,10,13]$. These estimates have been widely distributed and now frequently form the context for discussions among the media and other of the role of the Internet in health care. Other, less well-publicized reports suggest much lower rates of Internet use
$[2,9]$. In fact, little is known about to what extent patients in different countries access the Internet and whether they benefit from consulting it $[5,8]$.

Cardiovascular diseases are the leading cause of mortality in the industrialized world and interventional or operative procedures have been increasingly used to prevent cardiac damage. Recent innovations in cardiac surgery, 
e.g. minimally invasive or off-pump procedures, have significantly decreased morbidity and mortality rates among cardiopathic patients [6,17]. An increasing number of patients now undergo coronary surgery without cardiac arrest and the heart-lung-machine.

Despite the recent progression and the new achievements in this field, patients still remain to be informed about the indications, risks, benefits and outcome of cardiac surgery $[11,14]$. Unfortunately, the majority of cardiopathic patients and their families remain unaware of new innovative surgical techniques. Adequate information on the Internet could increase the patient's knowledge about these procedures, their applications and limitations $[3,12]$. We therefore focused our study at a midsize German cardiothoracic clinic in former Eastern Germany on three central research questions: Do cardiopathic patients and their families access the Internet to obtain healthrelated information? If this is the case, is online medical information understandable and useful for this non-medical public? Are there demographic and socioeconomic differences among internet and non-internet users?

\section{Methods}

Study sample

A prospective study among 255 patients, who underwent elective cardiac and thoracic operations was conducted between December 2003 and February 2004 at the Department of Cardiothoracic Surgery at the University Medical Centre Halle. Emergency patients and patients > 85 years were excluded from the study. A questionnaire comprising 25 questions was answered by the patients on the day of admission in the presence of a final year medical student. The student explained the questions and, if necessary, and the different meanings of the answers.

\section{Statistical analysis}

Data were collected in an Excel sheet. Discrete and continuous variables were analysed using the Chi-Square test, Fisher's exact test (where appropriate) or the Student ttest. Differences between variables were considered significant when $\mathrm{p}<0,05$. All calculations were performed with SPSS 11.0 [15] and StatsDirect 2.0.3 [16]. Data are presented either as mean \pm standard deviation or, where appropriate, as median and the $25^{\text {th }}$ and $75^{\text {th }}$ percentile, respectively.

\section{Results}

\section{Demographic data}

Two hundred fifty-five patients who met the inclusion criteria constituted the final sample. Mean age of the participants was $62,98 \pm 11,56$ years. Table 1 describes the sociodemographic characteristics of the sample more detailed. More than two-thirds of our participants were still married ( $\mathrm{n}=190,73,1 \%)$, twenty were divorced $(7,7$
$\%)$, seventeen were single $(7,5 \%)$ and twenty-four were widowed $(9,2 \%)$. On average, every patient lived with $2,11 \pm 0,88$ relatives at home (median 2,0$)$.

Considering Halle as a major German city with more than 220000 inhabitants, only 63 patients $(24,2 \%)$ came from Halle, the remaining lived in smaller cities and non-urban areas around Halle $(\mathrm{n}=192,75,8 \%)$. Table 2 summarizes the reasons for admission at our clinic.

\section{Prevalence and frequency of internet use}

Almost $70 \%$ of our participants did not own a personal computer $(\mathrm{n}=179)$, whereas twenty-one $(8,1 \%)$ had a PC, but no internet access, and fifty-five $(20,8 \%)$ had a computer with internet access. Interestingly, only $18 \%$ (n $=47$ ) used the internet, the remaining participants had never used the internet before. Thirty-six (13,8 \% of total) used the internet at home, seven $(2,7 \%)$ accessed it at the working place and four $(1,6 \%)$ went online in an internet café $(\mathrm{n}=1)$ or at friends/family $(\mathrm{n}=3)$. Among the internet users, there were six women and 41 men $(53,5 \pm 14,2$ versus $49,7 \pm 17,8$ years, $p=n . s$.). There were no significant differences between female and male users with

Table I: Sociodemographic characteristics of survey sample $(n=$ 255)

n (\%)

Age (years)
\[ \begin{array}{rc}20-49 & 26(10,2) \\ 50-64 & 94(36,8) \\ 65-80 & 132(51,8) \\ >80 & 3(1,2)\end{array} \]

Gender

$\begin{array}{rr}\text { female } & 69(27) \\ \text { male } & 186(73)\end{array}$

Education

$\begin{array}{rc}\text { no school } & 5(2) \\ 8^{\text {th }}-\text { class } & 132(52) \\ 10^{\text {th }}-\text { class } & 65(25,5) \\ \text { A-levels } & 9(3,5) \\ \text { university degree } & 44(17)\end{array}$

Profession

$\begin{array}{rc}\text { Senior citizen } & 182(7 \mathrm{I}) \\ \text { employees and manager } & 30(12) \\ \text { self-employed person } & 10(4) \\ \text { Student } & 2(0,8) \\ \text { worker } & 3(1,2) \\ \text { unemployed } & 28(11)\end{array}$

A-levels usually refers to the completion of twelve school years, which is prerequisite for attending a university in Germany. As males are affected more extensively by cardiovascular diseases than woman, hence our study population included more than twice the number of males than females. 
Table 2: Operation among the survey sample $(n=255)$

\begin{tabular}{lc}
\hline & $\mathrm{n}(\%)$ \\
\cline { 2 - 2 } Coronary artery bypass grafting (CABG) & $158(60,8)$ \\
Pacemaker/Defibrillator implantation & $16(6,2)$ \\
Cardiac valve replacement/reconstruction & $16(6,2)$ \\
Lung operation & $15(5,8)$ \\
Combination (CABG and valve) & $23(8,5)$ \\
Congenital defect repair & $1(0,4)$ \\
Other & $26(10)$ \\
\hline
\end{tabular}

respect to education, duration of internet use and computer possession.

Considering the monthly internet use, twenty-three $(8,8$ $\%$ ) internet users accessed the web more than 20 hours per month, eight $(3,1 \%)$ used it $10-20$ hours per month, eight 5 - 10 hours per month and the remaining eight participants went online less than 5 hours per month. We examined the reasons why and how often patients used the internet (Table 3). Only 17 respondents used the internet for health or health care information without a gender preference (2/6 women versus $15 / 41$ men, $\mathrm{p}=$ n.s.).

The reasons for not using the internet were as follows: disinterest $(n=136,52,3 \%)$, too complicate $(n=24,9,2 \%)$, too expensive $(n=21,8,1 \%)$, don't know $(n=3,1,2 \%)$.

Internet use and retrieval of relevant health information We also asked the patients whether or not they looked for information about her/his specific cardiac/thoracic disease and the planned operation at our hospital. Only 14 respondents $(5,4 \%)$ reported to have used the internet for retrieving specific information about cardiac or thoracic diseases. Interestingly, but not significantly, no female patients searched for specific disease information, whereas fourteen male patients went online to look for specific information $(\mathrm{p}=0,19)$. Additionally, the families or friends of 12 patients went online to find information on the specific conditions. Asked how relevant these information had been, respondents rated them on an analogous scale from 1 (not relevant) to 5 (very relevant) on median with 4,00 (range $3-4,5$ ). Nine of those who used the internet for health or health care information retrieval said, that internet information helped to better understand their symptoms and their planned treatment. Only one respondent found the information helpful to manage the own condition.

As cardiovascular diseases comprise the most popular and most cost-intensive disorders, we wanted to know, if patients use specific newsgroups or online chats. Only one internet user had ever tried to use these specific offer to find relevant information.

\section{Internet use is age and education dependent}

Internet users in our sample were significant younger than non-user (median $57(60,72)$ versus $67(47,63)$ years, p $<0,0001)$. Moreover, internet user had a higher education level. Nineteen of 47 users $(40,4 \%)$ had a university degree, $12,76 \%(n=6)$ had the A-levels and 16 patients (34\%) finished the $10^{\text {th }}$ class of a secondary school. The majority of non-users ( $\mathrm{n}=126,60,57 \%)$ had finished the $8^{\text {th }}$ class of a secondary school, 49 patients $(23,55 \%)$ finished the $10^{\text {th }}$ class of a secondary school, 5 did not attend any school, whereas only $12 \%(n=25)$ had a university degree.

\section{Impact of a specific web-based interface of our department}

As our clinic maintains an own website containing relevant information for our patients (e.g. information about specific operation procedures), we wanted to know, if and how our patients value electronic information technologies.

Asked if the patients had ever visited our department website, only five $(1,96 \%)$ respondents agreed, whereas the vast majority disagreed. As we plan to extend our electronic patient service, we asked how special services would be evaluated. First, we asked if an extended drug information service, e.g. a detailed explanation of certain

Table 3: Prevalence and Frequency of Internet Use ( $n=47$ internet users)

\begin{tabular}{|c|c|c|c|c|}
\hline How often did you & daily & I- 2/week & $I-2 /$ month & $I-2 / 3$ month \\
\hline use the internet for email $(n=34)$ & 17 & 14 & 2 & I \\
\hline use the internet for retrieving general information $(n=47)$ & 20 & 23 & 3 & I \\
\hline use the internet for information about health or health care $(n=17)$ & 1 & 6 & 7 & 3 \\
\hline use the internet for amusement $(n=29)$ & 4 & 11 & 10 & 4 \\
\hline use the Internet for shopping $(n=26)$ & 2 & 4 & 8 & 12 \\
\hline use the internet for chatting $(n=16)$ & I & 3 & 8 & 4 \\
\hline use the internet to communicate with other people in newsgroups $(n=5)$ & 0 & 3 & I & I \\
\hline use the internet to maintain your own homepage $(n=4)$ & 0 & 1 & 2 & I \\
\hline
\end{tabular}

The search for health or health care information has not been well established yet among our study sample. 
side and cross effects of drugs prescribed after surgery, would be useful. More than $75 \%$ of all respondents found such an offer not useful and irrelevant ( $\mathrm{n}=216,85 \%)$ and only 39 patients $(15 \%)$ would use such a service. Considering a postoperative intermittent telemedical monitoring of some basic cardiac functions, e.g. heart rhythm, four out of five patients $(\mathrm{n}=204,78,5 \%)$ would not participate in such a service. Even electronic pre- and postoperative scheduling for ambulant visits have not positively valued. Almost $90 \%(n=224)$ of our patients preferred phone calls and information letters to an email.

Asked which health/disease related themes might be worth an electronic information delivery by email or special sections on our website, 189 participants had no interest, whereas the small remaining minority would liked to be informed on several topics such as postoperative physical and sexual behaviour or advice for emergencies.

Sixty-three $(24,2 \%)$ of all respondents would use the web-based resource, 95 patients $(36,5 \%)$ would only use the already existing sources of information (e.g. physicians, pharmacies) and the remaining 97 respondents interestingly $(37,5 \%)$ found a printed brochure much more useful than any electronic resource.

In summary, our respondents valued electronic information on an analogous scale from 1 (not useful) to 5 (very useful) on median with 1,0. Asked if they would now visit our clinic website after answering the questionnaire, still $73,5 \%(\mathrm{n}=191)$ would not and only 63 patients $(24,2$ $\%)$ might visit our site to find useful information.

\section{Discussion}

Current estimates suggest that an increasing number of people access the World Wide Web and at least $2 \%$ out of more than 3 billion websites are assessed to be health related $[1,10]$. However, many patients that could actually benefit from proper use of the World Wide Web are unfamiliar with the new computer technologies and in the majority of cases are not even internet users. A recent report from the German Federal Statistic Office showed that Internet use varies widely within Europe, North America and Asia and represents a highly dynamic market with rapidly growing numbers of users. In 2002, $44 \%$ of German households had internet access, whereas e.g. 66 $\%$ of all Dutch households and $51 \%$ of all US-households were equipped with Internet access [18]. In Spain and Japan, in contrast, $30 \%$ and $35 \%$, respectively, of all households had Internet access. Only $21 \%$ of patients in our sample possessed a PC with internet access and only $18 \%$ of respondents used the Internet regularly. Internet use is age, income and education dependent [1] and our data are consistent with these findings. The older people are, the less frequent the internet access is. Forty-one percent of all Germans between 55 - 64 years have a PC with internet access, whereas this number decreases to $20 \%$ in the group of all 65 - 74 years old Germans and drops to $12 \%$ in the group of the 75 years and older Germans. Having a personal computer with internet access does not imply that the internet is used regularly. Only $8 \%$ of all Germans older than 65 years and $27 \%$ between $55-64$ years used the internet in 2003. Among the European Community, internet use in Germany ranks far behind Scandinavia and Great Britain [18].

The reasons for the low PC and internet use in our study sample below the German and European average might be due to differences in the social structure between Western- and Eastern Germany such as the higher unemployed rate and the higher percentage of retired people and a less frequent internet usage in these groups compared to employees and students [18]. Furthermore, the internet users in our sample were significantly more educated than non-users and achieved higher academic degrees.

Although an increasing number of health-related websites became accessible, only 14 patients in our sample used this service. Health information retrieval played only a minor role for the internet users in our sample population. A recently conducted survey among German households showed that $24 \%$ of Eastern German internet users search for health information online, in contrast to $35 \%$ of Western Germans. The reasons for this difference have not been elucidated yet. One possible reason might be the latent fear in former Eastern Germany for potential misinformation and incredibility present in unprofessional websites and the underdeveloped contact to websites developed by the immediate health-care provider. We also believe that there is a growing need for objective, reproducible, widely accepted criteria that can form the basis for regulation of publication of medical material on the internet [7].

The interest in an extensive health-related internet use of our study sample is quite low. Although those, who went online for health information retrieval, valued the received information positively, other resources such as newsgroups, self-help groups, chat rooms and even visiting our clinic homepage, where several additional services are offered, have been fairly unknown among our study sample. There is a clear demand for continuing education, especially for senior people, to facilitate the access to the growing, modern and indispensable medium internet. Despite all the advantages the internet offers, printed media and personal contacts still proved to be the most reliable source of information among our study sample. 


\section{Study limitations}

This study is limited to one medium-sized Eastern German university with its specific demographic and social conditions. Since Halle with its strong industrial background before reunification and nowadays a high rate of unemployment and retired people represents a typical former Eastern German industrial city, we probably underestimate the real prevalence and frequency of internet use in our study compared to other areas in Western Germany.

\section{Conclusion}

Continuing education, particularly for the elderly, remains necessary to facilitate the computer and internet use in former Eastern Germany. The advantages of electronic web-based media and professional health-care websites have not been fully employed by our sample yet. Conversely, web-based health care resources would be used more extensively, if adequate information was available to the patients.

\section{Competing interests}

The author(s) declare that they have no competing interests.

\section{Authors' contributions}

DS examined and selected the study patients. He explained the questionnaire to the patients and collected the data in a database. He also was involved in the coordination and interpretation of the data.

CD designed the study, developed the questionnaire, performed the statistical analysis and drafted the manuscript and revised it in cooperation with the co-author.

\section{Acknowledgements}

We would like to appreciate the help of Professor Dr. Rolf-Edgar Silber, who, as the department chair, supported this study in general and revised critically the draft.

\section{References}

I. Fox S, Rainie L, Horrigan J, Lenhart A, Spooner T, Burke M, Lewis O, Carter C: The online health care revolution: How the Web helps Americans take better care of themselves. Pew Internet and American Life Project 2000.

2. Brodie M, Flournoy RE, Altman DE, Blendon RJ, Benson JM, Rosenbaum MD: Health information, the Internet, and the digital divide. Health Aff (Millwood) 2000, 19:255-265.

3. Powell J, Clarke A: The WWW of the World Wide Web: Who, What, and Why? J Med Internet Res 2002, 4:e4.

4. Fogel J, Albert SM, Schnabel F, Ditkoff BA, Neugut Al: Use of the Internet by women with breast cancer. J Med Internet Res 2002, 4:E9.

5. Benigeri M, Pluye P: Shortcomings of health information on the Internet. Health Promot Int 2003, I 8:38I-386.

6. Eysenbach G, Kohler C: How do consumers search for and appraise health information on the world wide web? Qualitative study using focus groups, usability tests, and in-depth interviews. Bmj 2002, 324:573-577.
7. Cline RJ, Haynes KM: Consumer health information seeking on the Internet: the state of the art. Health Educ Res 200I, | 6:67|-692.

8. D'Ancona G, Karamanoukian HL, Soltoski P, Salerno TA, Bergsland J: Changing referral pattern in off-pump coronary artery bypass surgery: a strategy for improving surgical results. Heart Surg Forum 1999, 2:246-249.

9. Tang LW, D'Ancona G, Bergsland J, Kawaguchi A, Karamanoukian HL: Robotically assisted video-enhanced-endoscopic coronary artery bypass graft surgery. Angiology 2001, 52:99-102.

10. Scherrer-Bannerman A, Fofonoff D, Minshall D, Downie S, Brown M, Leslie F, McGowan P: Web-based education and support for patients on the cardiac surgery waiting list. J Telemed Telecare 2000, 6 Suppl 2:S72-4.

II. Leaffer T, Gonda B: The Internet: an underutilized tool in patient education. Comput Nurs 2000, 18:47-52.

12. Murero M, D'Ancona G, Karamanoukian H: Use of the Internet by patients before and after cardiac surgery: telephone survey. J Med Internet Res 200I, 3:E27.

13. Brennan PF, Caldwell B, Moore SM, Sreenath N, Jones J: Designing HeartCare: custom computerized home care for patients recovering from CABG surgery. Proc AMIA Symp 1998:38I-385.

14. SPSS: SPSS-Website. [http://www.systat.com].

15. StatsDirect: StatsDirect-Website. [http://www.statsdirect.com].

16. Baker L, Wagner TH, Singer S, Bundorf MK: Use of the Internet and e-mail for health care information: results from a national survey. Jama 2003, 289:2400-2406.

17. Timm U: Information Technology in German Households. Results from a pilot Study in 2003. Wiesbaden, Germany, Federal Bureau of Statistics; 2004: I-48.

18. Eysenbach G: Issues in evaluating health websites in an Internet-based randomized controlled trial. J Med Internet Res 2002, 4:EI7.

\footnotetext{
Publish with Biomed Central and every scientist can read your work free of charge

"BioMed Central will be the most significant development for disseminating the results of biomedical research in our lifetime. "

Sir Paul Nurse, Cancer Research UK

Your research papers will be:

- available free of charge to the entire biomedical community

- peer reviewed and published immediately upon acceptance

- cited in PubMed and archived on PubMed Central

- yours - you keep the copyright

Submit your manuscript here:

http://www.biomedcentral.com/info/publishing_adv.asp

BiolMedcentral
} 